\title{
The Effect of Gestational Weight Gain Across Reproductive History on Maternal Body Mass Index in Midlife: The Study of Women's Health Across the Nation
}

\author{
Franya Hutchins, MS, ${ }^{1}$ Barbara Abrams, DrPH, ${ }^{2}$ Maria Brooks, $\mathrm{PhD},{ }^{1}$ Alicia Colvin, $\mathrm{PhD},{ }^{1}$ \\ Tiffany Moore Simas, MD, ${ }^{3}$ Milagros Rosal, $\mathrm{PhD},{ }^{4}$ Barbara Sternfeld, $\mathrm{PhD},{ }^{5}$ and Sybil Crawford, $\mathrm{PhD}^{6}$
}

\begin{abstract}
Background: Excessive weight gain during pregnancy is common and has been shown to be associated with increased long-term maternal weight. However, less is known on whether there is a cumulative effect of excessive gestational weight gain (GWG) over multiple pregnancies.

Methods: Data from the Study of Women's Health Across the Nation were used, restricted to parous women with no history of stillbirth or premature birth. The effect of the number of excessive GWG pregnancies on body mass index (BMI) in midlife (age 42-53) was analyzed using multivariable linear regression. Fully adjusted models included parity, inadequate GWG, demographic, and behavioral characteristics.

Results: The 1181 women included in this analysis reported a total of 2693 births. Overall, 466 (39.5\%) were categorized as having at least one pregnancy with excessive GWG. The median BMI at midlife was $26.0 \mathrm{~kg} / \mathrm{m}^{2}$ (interquartile range 22.5-31.1). In fully adjusted models, each additional pregnancy with excessive GWG was associated with 0.021 higher estimated $\log$ BMI $(p=0.031)$. Among women with $1-3$ births, adjusted mean (95\% confidence interval) BMI for those with $0,1,2$, and 3 excessive GWG pregnancies was 25.4 (24.9-25.9), 26.8 (26.1-27.5), 27.5 (26.6-28.4), and 28.8 (27.3-30.5), respectively.

Conclusions: In this multiethnic study of women with a history of term live births, the number of pregnancies with excessive GWG was associated with increased maternal BMI in midlife. Our findings suggest that prevention of excessive GWG at any point in a woman's reproductive history can have an impact on long-term maternal health.
\end{abstract}

Keywords: gestational weight gain, pregnancy, obesity, race and ethnicity, body mass index, midlife

\section{Introduction}

M ORE THAN ONE in three adults in the United States have obesity, with the highest prevalence among women in midlife. ${ }^{1}$ Those with obesity are at an elevated risk of adverse health outcomes, including type 2 diabetes and fatal coronary heart disease. ${ }^{2}$ Prevention of obesity is complicated by social, behavioral, and biological factors. ${ }^{3}$ Therefore, the identification of modifiable risk factors is a key public health concern.
A potential opportunity for obesity prevention before midlife is during pregnancy. Excessive gestational weight gain $(\mathrm{GWG})$ is common ${ }^{4}$ and is associated with long-term weight. ${ }^{5}$ Findings from cohort studies suggest that over $40 \%$ of pregnant women exceed clinical guidelines for GWG adequacy. ${ }^{6,7}$ This can have a long-term impact on maternal health. Excessive GWG has been linked to increased weight ${ }^{8}$ and higher odds of obesity ${ }^{9,10}$ over 5 years after pregnancy. Some evidence supports an association remaining more than 15 years. ${ }^{11,12}$

\footnotetext{
${ }^{1}$ Department of Epidemiology, Graduate School of Public Health, University of Pittsburgh, Pittsburgh, Pennsylvania.

${ }^{2}$ Division of Epidemiology, School of Public Health, University of California, Berkeley, Berkeley, California.

Departments of ${ }^{3}$ Obstetrics and Gynecology and ${ }^{4}$ Quantitative Health Sciences, University of Massachusetts Medical School, Worcester, Massachusetts.

${ }^{5}$ Division of Research, Kaiser Permanente, Oakland, California.

${ }^{6}$ Department of Medicine, University of Massachusetts Medical School, Worcester, Massachusetts.
} 
While the current literature consistently shows a positive association of excessive GWG in a single pregnancy with maternal weight later in life, the impact of GWG over a woman's entire reproductive history is not well understood. Few studies have considered whether weight characteristics across multiple pregnancies have a cumulative effect on maternal health. ${ }^{12,13}$ None to our knowledge has estimated the effect of GWG adequacy in all of a woman's pregnancies, while controlling for lifestyle factors. The Study of Women's Health Across the Nation (SWAN) provides an opportunity to incorporate the full reproductive history of participants. The 22-year prospective SWAN study has been an important source of data on the menopausal transition and reproductive health. ${ }^{14-16}$ It is one of the few cohort studies that captured lifetime reproductive history in U.S. women. The goal of this analysis was to evaluate how GWG adequacy across all of a woman's pregnancies contributed to obesity in midlife among primiparous and multiparous SWAN participants.

\section{Methods \\ Participants}

SWAN cohort. This analysis involves a subset of women participating in SWAN, which is an ongoing prospective, multiethnic multicenter study of women over the menopausal transition. The SWAN cohort is made up of 3302 women enrolled from 7 cities: Boston, MA, Chicago, IL, Detroit, MI, Los Angeles, CA, Oakland, CA, Newark, NJ, and Pittsburgh, PA. Enrollment began in 1996 with the following primary eligibility criteria: age $42-52$, having at least one menstrual period in previous 3 months, no exogenous hormone use in previous 3 months, intact uterus, at least one ovary, and self-identification with a designated racial/ethnic group recruited by site. All sites enrolled women who identified as non-Hispanic white as well as women from one additional race or ethnic group: NonHispanic black, Hispanic, Japanese, or Chinese. More information on the sampling strategy for SWAN has been published previously. ${ }^{17}$ IRB approval was obtained with each site institution and written consent given by all participants.

Data collection. A comprehensive reproductive history questionnaire was administered at the 13th follow-up visit (conducted in 2011-2013), which included prepregnancy weight, GWG amount, and gestational age for each live birth. Although SWAN also collected GWG amount for each pregnancy at the baseline visit (conducted in 1996-1997), prepregnancy weight and gestational age were not assessed at that time and all reproductive characteristics in our primary analysis were obtained from the visit 13 questionnaire.

Analytic sample. The goal of this analysis was to evaluate the association between excessive GWG and midlife obesity among women with a history of term, singleton live births. Therefore, the analytic sample was drawn from women who reported one or more live births at term in their lifetime $(n=2732)$. Women with a history of anorexia, bulimia, or thyroid disorders $(n=303)$ were excluded.

Of the 2429 women reporting live birth(s) with no history of disordered eating or thyroid disease, 1639 completed a reproductive history questionnaire at SWAN follow-up visit 13. Those with multigestational births or pregnancies that lasted 5 months or longer resulting in stillbirth or premature birth were excluded $(n=263)$. In addition, women with missing outcome data $(n=14)$, missing prepregnancy, or gestational weight data for more than one pregnancy or for their only pregnancy if primiparous $(n=73)$, as well as one participant who reported a birth after the outcome of midlife body mass index (BMI) was measured were excluded. Two additional women who reported GWG outside likely biological plausibility as defined in previous literature were excluded. ${ }^{18,19}$ In sensitivity analyses, participants with missing covariate values $(n=105)$ were included using mean imputation. Estimates for main predictors did not differ meaningfully between the complete case and imputed models (results not shown). Results presented are from complete case analyses. The final analytic sample included 1181 women representing 2693 singleton, term births. Figure 1 outlines participant eligibility and exclusions.

\section{Measures}

Exposure. Prepregnancy weight and pregnancy weight gain amount for each live birth reported were collected by retrospective self-report at visit 13 (when women ranged in age from 56 to 68). Interviews were conducted in English, Spanish, Cantonese, and Japanese depending on site and participant. Prepregnancy BMI was calculated with the retrospective prepregnancy weight collected at visit 13 and height measured at baseline (visit 0). Adequacy of GWG, the primary exposure, was calculated for each pregnancy based on the Institute of Medicine's 2009 guidelines ${ }^{20}$ and characterized as "inadequate," "adequate," or "excessive" as per prepregnancy BMI category (Supplementary Table S1). The Institute of Medicine guidelines represent the current state of knowledge on the impact of GWG on infant and maternal health. Because these are the most clinically relevant today, we applied the 2009 recommendations in this analysis as opposed to prior standards.

Alternative BMI cutoffs were used for Japanese and Chinese participants, with overweight defined as $\geq 23 \mathrm{~kg} / \mathrm{m}^{2}$ and obese as $\geq 25 \mathrm{~kg} / \mathrm{m}^{2}$. This is consistent with recommendations from the Western Pacific Region WHO and based on literature demonstrating higher body fat in Asians at lower BMI values compared to European heritage populations. ${ }^{21}$ Prior research supports these cutoffs for Japanese and Chinese populations living in Asia ${ }^{22-25}$ and people of Asian heritage living in North America. ${ }^{26,27}$ No adjustments were made to GWG adequacy cut points as the IOM recommends these guidelines across racial/ethnic groups. Recent research has supported use of the IOM guidelines to predict maternal outcomes in Asian and Asian American populations. ${ }^{28,29}$

Outcome. The primary outcome was midlife BMI $\left(\mathrm{kg} / \mathrm{m}^{2}\right)$, calculated from weight and height measured at the SWAN baseline visit by trained staff according to a standard protocol when women ranged in age from 42 to 53 years. Obesity was defined as BMI $\geq 30 \mathrm{~kg} / \mathrm{m}^{2}$ for white, black, and Hispanic women and BMI $\geq 25 \mathrm{~kg} / \mathrm{m}^{2}$ for Japanese and Chinese women as noted above.

Covariates. Covariates collected at the baseline visitconcurrent with the outcome assessment—were age (years), race/ethnicity (non-Hispanic black, Chinese, Japanese, Hispanic, and non-Hispanic white), education level (categorized 


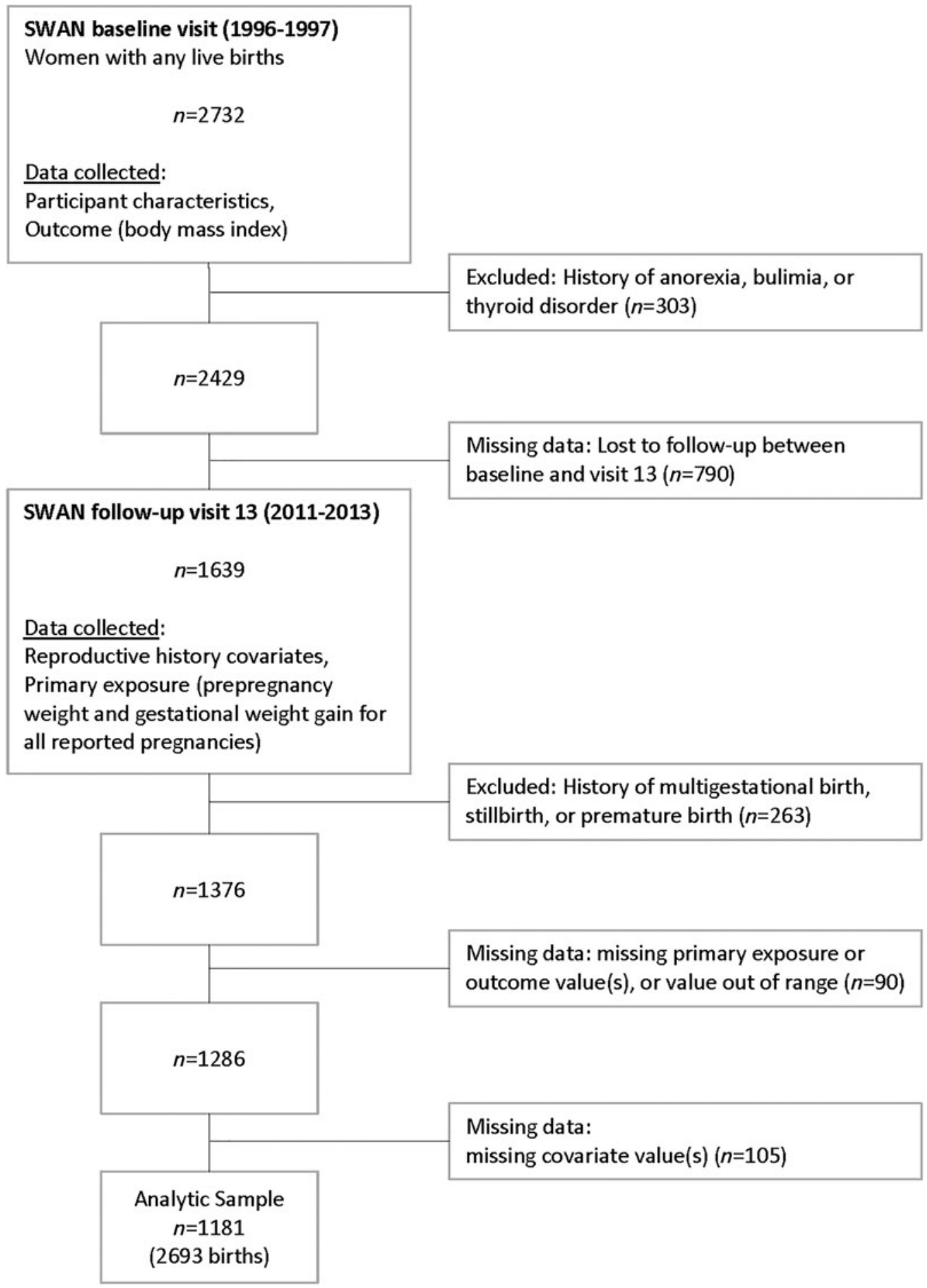

FIG. 1. Participant and data collection flowchart.

as high school or less, some college/college degree, or postcollege study), age at first pregnancy (years), time since last pregnancy (years), smoking status at midlife (current, previous, or never smoker), adolescent BMI $\left(\mathrm{kg} / \mathrm{m}^{2}\right)$, difficulty in paying for basics (somewhat hard/very hard, or not very hard), menopausal status (premenopause or early perimenopause), daily caloric intake (kcal), physical activity (score), and stress level (score). Study site was also included as a covariate.
Adolescent BMI was collected by participant recall of high school weight. Menopausal status was assessed by self-report of bleeding over the 12 months before study visit. Caloric intake at midlife was calculated from responses to a modified Block interviewer-assisted food frequency questionnaire completed at the SWAN baseline visit. ${ }^{30}$ The total physical activity score is derived from an adaptation of the Kaiser Permanente Health Plan Activity Survey used previously by SWAN investigators. ${ }^{31}$ The score ranges from 1 to 15 and 
represents the sum of responses to 11 physical activity questions covering sports activities, nonsport leisure activities, and household/childcare activities. Stress is represented by a score summing the frequency of feeling overwhelmed during the past 2 weeks $(1=$ Never to $5=$ Very Often $)$ based on four component questions in the SWAN screener. Scores range from 4 (low stress) to 20 (high stress).

Additional reproductive history characteristics retrospectively self-reported at visit 13 were parity, number of pregnancies with a gestational hypertensive disorder, and number of pregnancies with gestational diabetes.

\section{Statistical analysis}

Data transformations. The outcome measure BMI was transformed by natural log when treated continuously to account for skewed distribution.

Descriptive statistics for participant characteristics. We summarized participant characteristics for the full analytic sample and stratified by whether a woman had ever experienced a pregnancy with excessive GWG. Categorical characteristics are presented by number and percent of women with differences evaluated by chi-square test. Continuous variables with approximately normal distributions are presented by mean and standard deviation, and compared by two-sided $t$-tests. Adolescent and midlife BMI are presented by median and interquartile range with two-sided Wilcoxon $p$-values.

Modeling midlife BMI. The primary exposure was the number of pregnancies in a woman's life with excessive GWG. We used nonparametric locally weighted scatterplot smoothing (loess) regression on number of excessive GWG pregnancies to identify the functional form of the association with midlife BMI. ${ }^{32}$ The association between number of pregnancies with excessive GWG per woman and midlife log BMI was estimated by linear regression. A priori hypotheses of interaction between excessive GWG with parity and with race/ethnicity were tested in separate fully adjusted models.

Unadjusted, minimally adjusted, and fully adjusted results are reported. Modeling the outcome as log-transformed BMI best fits the distribution of the data and demonstrates the attenuation of effect with the addition of covariates across models. To provide a clinically relevant interpretation, adjusted means of $\log$ BMI were estimated for women with 1-3 births ( $n=1027)$ by treating number of excessive GWG pregnancies categorically. Adjusted means were back-transformed to the original scale for presentation. Bonferroni-adjusted $p$-values were calculated for pairwise group comparisons.

Modeling odds of midlife obesity. Odds ratios (ORs) and 95\% confidence intervals (CIs) for obesity at midlife were estimated by logistic regression using the number of excessive GWG pregnancies as a continuous predictor. To assess the potential impact of the timing of excessive GWG across pregnancies, ORs and 95\% CIs for obesity were also estimated by logistic regression using the GWG adequacy of each woman's last birth as a categorical predictor (among women with one to three births).
Reliability of the GWG measure. To evaluate the reliability of the primary exposure variable-self-reported GWG-the difference between the GWG reported at the study baseline visit and at follow-up visit 13 was calculated for each pregnancy. Mean and standard deviation of these differences as well as Spearman correlations between the two GWG amounts are reported stratified by birth number. We tested whether the difference between the two GWG values varied by midlife obesity status with two-sample $t$-tests. Because preliminary results indicated poorer correlation for GWG amounts reported on the fifth or higher birth, sensitivity analyses were performed for the fully adjusted linear regression models of midlife BMI by excluding the $n=13$ women with $\geq 5$ births.

All analyses were performed using SAS v. 9.4 (SAS Institute, Cary, NC).

\section{Results}

\section{Participant characteristics}

In total, 985 women were excluded from the analytic sample for missing data. These participants were different from the women included in this analysis in several ways, including race/ethnicity and educational attainment (Supplementary Table S2).

Table 1 shows participant characteristics by excessive GWG status. Of the 1181 SWAN participants included, 715 $(60.5 \%)$ women reported having no pregnancy with excessive GWG and 466 (39.5\%) reported one or more. Supplementary Table S3 presents a cross tabulation of number of excessive GWG pregnancies by parity. Participants reported up to 8 births, with a mean parity of 2.3 births. Parity did not differ significantly by whether a woman had any excessive GWG pregnancies. The mean time between the last birth and the outcome measure of midlife BMI was $15.0 \pm 6.73$ years.

Women who had ever experienced an excessive GWG pregnancy - compared to no excessive GWG pregnancieswere more likely to be black and less likely to be Japanese than white. In addition, women who had one or more excessive GWG pregnancies reported higher mean adolescent BMI, were younger at first pregnancy, were less likely to be never smokers, and had a lower mean physical activity score at midlife in unadjusted comparisons. The mean age at the outcome time point was $46.6 \pm 2.64$ years. The median midlife BMI was $26.0 \mathrm{~kg} / \mathrm{m}^{2}$, and nearly one-third of participants had an obese BMI (32.7\%). Median midlife BMI was 4.4 units higher among those who reported one or more excessive GWG pregnancies compared to those who did not $(p<0.001)$. The prevalence of obesity at midlife was also higher among those with one or more excessive GWG pregnancies $(47.6 \%)$ compared to those with none (22.9\%, $p<0.001)$.

\section{Modeling midlife BMI}

A loess plot indicated an increase in BMI with each additional excessive GWG pregnancy (Fig. 2). A change in slope after the first pregnancy with excessive GWG suggested a difference in the effect between none and any compared to the overall number of such pregnancies. To address this, we included a dichotomous term representing no excessive GWG pregnancies (coded as 0 ) versus one or more 
Table 1. Participant Characteristics at Time of Outcome Assessment

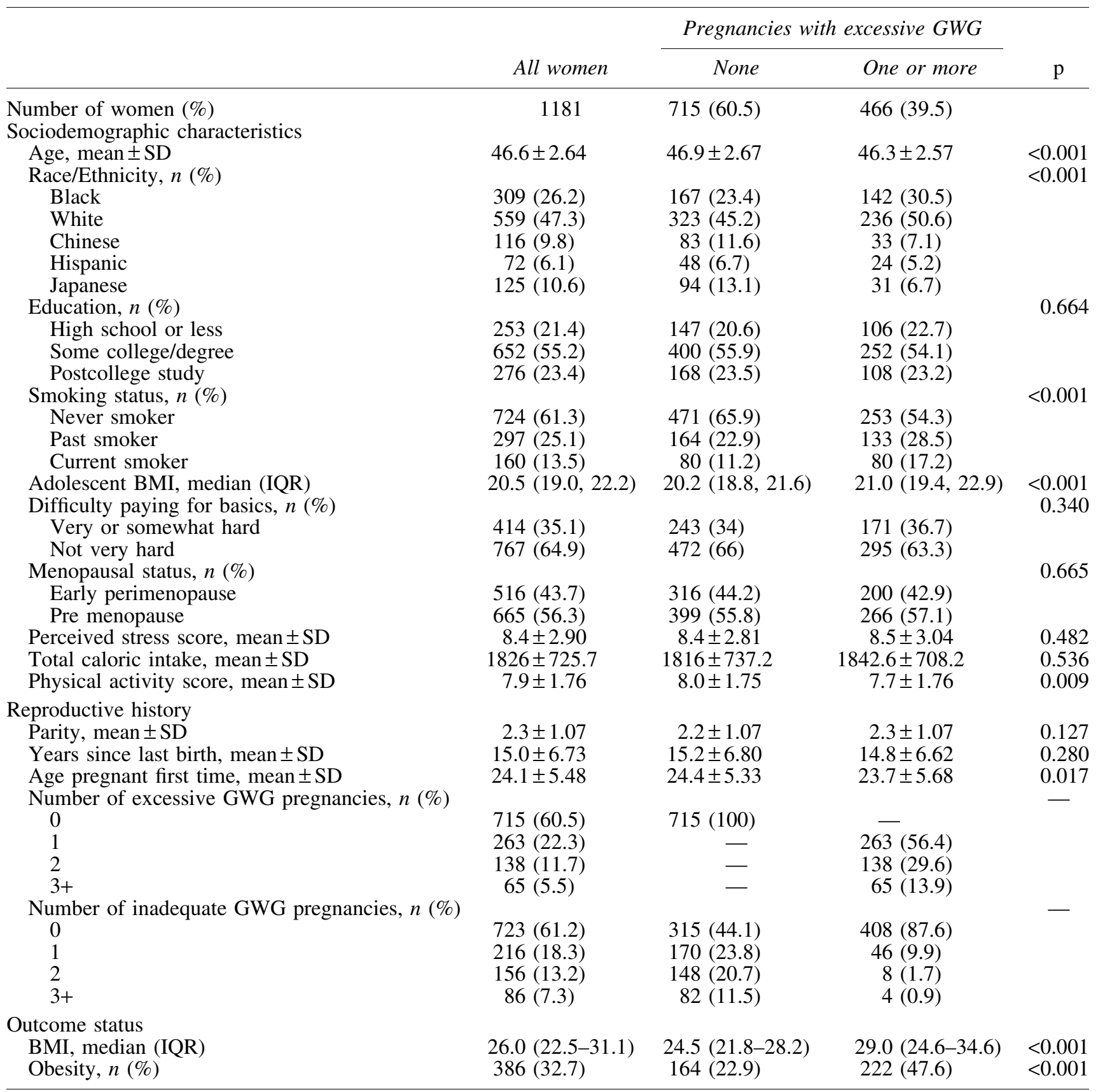

BMI, body mass index; GWG, gestational weight gain; IQR, interquartile range; SD, standard deviation.

(coded as 1). This adjustment accounts for the piecewise nature of the slope.

Each excessive GWG pregnancy was associated with an increase of $0.078 \log$ BMI units in the unadjusted model (Table 2). This association was attenuated to a coefficient of $0.020 \log$ BMI units in the minimally adjusted model, but remained statistically significant. Addition of the potential mediating variables-number of pregnancies with hypertensive disorder and number with gestational diabetes-did not substantially change the estimated effect size or $p$-value for the main exposure. Hypertensive disorder was a significant, independent predictor of midlife BMI $(p=0.001)$, while gestational diabetes was not $(p=0.270)$. The interac- tion terms tested between excessive GWG with parity and with race/ethnicity were not significant $(p>0.7)$ and were not included in final models (results not shown). Potential collinearity of predictor variables was evaluated by variance inflation factor and determined not to be present (all values of VIF were $<4$, results not shown).

Women with excessive GWG pregnancies had a higher midlife mean BMI compared to those with none (Table 3). Women with no excessive GWG had an adjusted marginal mean BMI of $25.4 \mathrm{~kg} / \mathrm{m}^{2}$ at midlife $(95 \% \mathrm{CI}=24.9-25.9)$ compared to a BMI of $28.8 \mathrm{~kg} / \mathrm{m}^{2}$ for those reporting excessive GWG in three pregnancies $(95 \% \mathrm{CI}=27.3-30.5)$. The adjusted mean BMI values for each increase from 1 to 3 


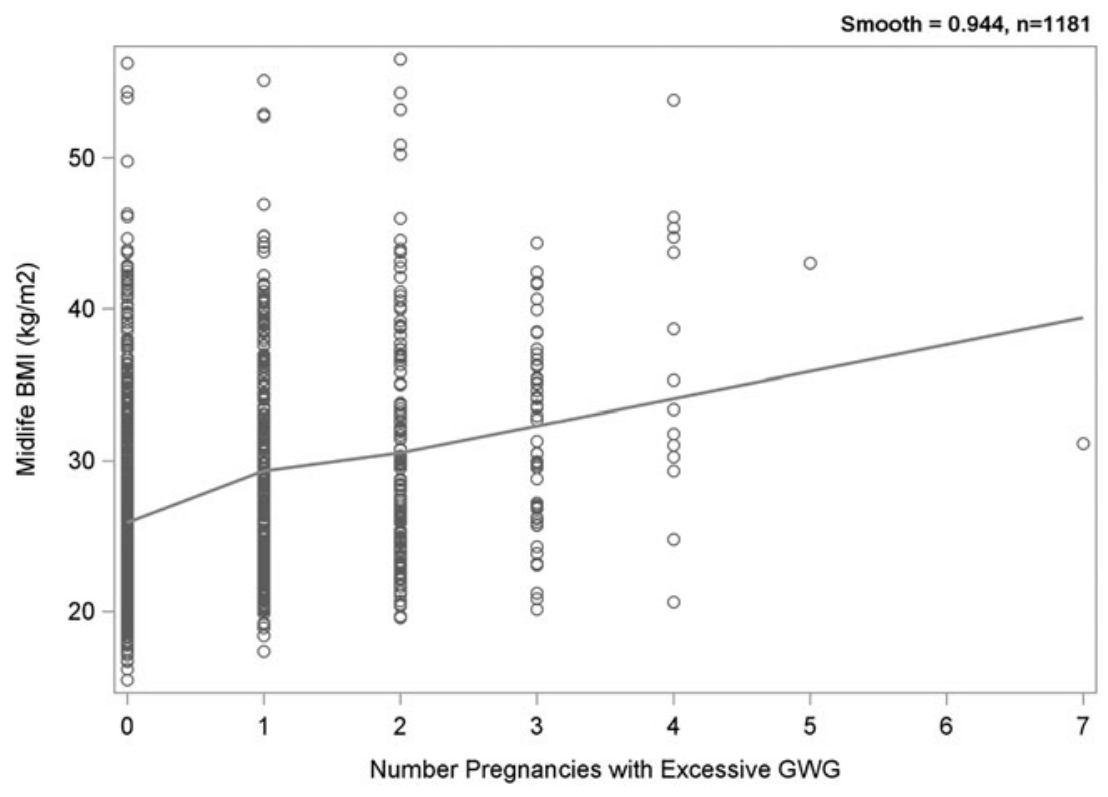

FIG. 2. Loess plot of midlife BMI by number of excessive GWG pregnancies. BMI, body mass index; GWG, gestational weight gain.
Table 2. Change in Log-Transformed Body Mass Index at Midlife PER Number of PREgnancies with Excessive Gestational Weight Gain

\begin{tabular}{|c|c|c|c|}
\hline Predictor & Slope & $S E$ & $\mathrm{p}$ \\
\hline \multicolumn{4}{|l|}{ Model 1, Unadjusted } \\
\hline $\begin{array}{l}\text { Number of excessive GWG } \\
\text { pregnancies }\end{array}$ & 0.078 & 0.007 & $<0.001$ \\
\hline \multicolumn{4}{|l|}{ Model 2, Minimally adjusted ${ }^{\mathrm{a}}$} \\
\hline $\begin{array}{l}\text { Number of excessive GWG } \\
\text { pregnancies }\end{array}$ & 0.020 & 0.010 & 0.036 \\
\hline $\begin{array}{l}\text { Number of inadequate GWG } \\
\text { pregnancies }\end{array}$ & -0.012 & 0.005 & 0.030 \\
\hline $\begin{array}{l}\text { Any excessive GWG pregnancy } \\
(0 / 1)\end{array}$ & 0.048 & 0.018 & 0.008 \\
\hline Parity & 0.009 & 0.006 & 0.143 \\
\hline \multicolumn{4}{|l|}{ Model 3, Fully adjusted ${ }^{\mathrm{b}}$} \\
\hline $\begin{array}{l}\text { Number of excessive GWG } \\
\text { pregnancies }\end{array}$ & 0.021 & 0.010 & 0.031 \\
\hline $\begin{array}{l}\text { Number of inadequate GWG } \\
\text { pregnancies }\end{array}$ & -0.011 & 0.005 & 0.042 \\
\hline $\begin{array}{l}\text { Any excessive GWG pregnancy } \\
(0 / 1)\end{array}$ & 0.044 & 0.018 & 0.015 \\
\hline Parity & 0.008 & 0.006 & 0.193 \\
\hline $\begin{array}{l}\text { Number of pregnancies with } \\
\text { hypertensive disorder }\end{array}$ & 0.044 & 0.014 & 0.001 \\
\hline $\begin{array}{l}\text { Number of pregnancies with } \\
\text { gestational diabetes }\end{array}$ & 0.020 & 0.019 & 0.270 \\
\hline
\end{tabular}

${ }^{\mathrm{a}}$ Model 2 adjusted for variables shown as well as study site, age at outcome measure, race/ethnicity, education, smoking, adolescent BMI, difficulty paying for basics, menopausal status, stress score, caloric intake, physical activity score, years since last birth, and age first pregnant.

${ }^{\mathrm{b}}$ Model 3 adjusted for variables noted with Model 2 as well as number of pregnancies with hypertensive disorder and number of pregnancies with gestational diabetes.

SE, standard error. excessive GWG pregnancies show a statistically significant monotonic relationship.

\section{Modeling odds of midlife obesity}

Each additional pregnancy with excessive GWG was associated with a $64 \%$ increase in odds of obesity at midlife $(\mathrm{OR}=1.64,95 \% \mathrm{CI}=1.20-2.25)$ in the fully adjusted model $(p=0.002$, see Fig. 3 and Supplementary Table S4). We also evaluated the relationship between GWG adequacy in the last pregnancy and odds of midlife obesity for those with one to three births (Fig. 4 and Supplementary Table S5). Among women with one birth, having excessive GWG in their pregnancy was not significantly associated with odds of obesity at midlife $(p=0.719)$. Among women with two births, the association between excessive GWG in the second pregnancy and obesity was nonsignificant $(p=0.185)$, although suggestive of increased odds with an OR of 1.78 (95\% $\mathrm{CI}=0.93-3.40$ ). Excessive GWG in the third of three births was significantly associated with 3.5 times higher odds of obesity at midlife $(\mathrm{OR}=3.54,95 \% \mathrm{CI}=1.37-9.14, p=0.027)$ in fully adjusted models, including adjustment for the GWG adequacy of the first and second pregnancies.

\section{Reliability of GWG measure}

The mean differences and Spearman correlations of selfreported GWG amount collected at the baseline visit and again at follow-up 13 for each birth are presented in Supplementary Table $S 6$. The mean difference ranged from a low of 1.8 pounds for the first birth to a high of 9.0 pounds for the sixth birth. Self-report in this cohort showed moderate reliability for the first four births reported, with Spearman $R^{2}$ values ranging from 0.73 for the first birth to 0.57 for the fourth birth. Correlation of GWG amount per birth was significant $(p<0.001)$ for the first four births reported, but not for the fifth birth and beyond.

The mean difference in GWG amounts reported, stratified by birth number, did not differ by midlife obesity status (Satterthwaite $p$-value $>0.17$ per birth). However, the variability of 
Table 3. Adjusted Marginal Mean Body Mass INDEX IN MidLIFE BY NuMber OF PREgNANCIES with Excessive Gestational Weight Gain, Among Women With One to Three BirThs

\begin{tabular}{lc}
$\begin{array}{l}\text { Number of } \\
\text { excessive GWG } \\
\text { pregnancies }\end{array}$ & $\begin{array}{c}\text { LS mean } \\
\text { BMI }(95 \% C I)^{\mathrm{a}}\end{array}$ \\
\hline $0(n=625)$ & $25.35(24.85-25.85)$ \\
$1(n=233)$ & $26.80(26.11-27.51)$ \\
$2(n=130)$ & $27.46(26.59-28.36)$ \\
$3(n=39)$ & $28.83(27.25-30.50)$ \\
\hline
\end{tabular}

${ }^{\mathrm{a}}$ Data are least squares mean BMI from fully adjusted model. Model adjusted for number of pregnancies with inadequate GWG, parity, study site, age at outcome measure, race/ethnicity, education, smoking, adolescent BMI, difficulty paying for basics, menopausal status, stress score, caloric intake, physical activity score, years since last birth, age first pregnant, number of pregnancies with hypertensive disorder, and number of pregnancies with gestational diabetes.

CI, confidence interval.

mean difference was greater among women with an obese midlife BMI compared to those without (equality of variance $F$-test $p$-values $<0.01$ per birth). Linear regression models of log-transformed BMI that excluded women with five or more births showed similar results to those of the primary analysis (Supplementary Table S7). In fully adjusted models, excluding women with five or more births strengthened the association between the number of excessive GWG pregnancies and midlife BMI $(\beta=0.025 \operatorname{logBMI}, p=0.017)$, and attenuated the effect of having any versus no excessive GWG pregnancies $(\beta=0.036 \log \mathrm{BMI}, p=0.062)$.

\section{Discussion}

In this multiethnic cohort of parous women, $39.5 \%$ reported GWG that exceeded IOM recommendations in at least one pregnancy, consistent with prior observations. ${ }^{4,6,7}$ The outcome of midlife obesity was experienced by $32.7 \%$ of women, measured at an average age of 46.6 \pm 2.64 . Nearly half $(47.6 \%)$ of women with excessive GWG had an obese BMI at midlife, compared to $22.9 \%$ of those who had never experienced excessive GWG. Our analysis found that each pregnancy with excessive GWG in a woman's life was associated with a $64 \%$ increase in the odds of obesity at midlife.
This association did not vary by race/ethnicity and was independent of factors including parity, years since last birth, and physical activity level. Finally, results comparing the odds of obesity by GWG adequacy in a woman's last pregnancy were inconclusive. This suggests that the total number of excessive GWG pregnancies may have more influence on maternal weight than the order of GWG adequacy across pregnancies.

To the current literature, our study adds evidence of a cumulative effect of multiple pregnancies with excessive GWG on long-term maternal health, independent of parity. Beyond the impact of ever versus never experiencing an excessive GWG pregnancy, each additional excessive GWG pregnancy contributed to higher mean midlife BMI. Notably, we demonstrated that this association extends to women of Japanese and Chinese ethnic backgrounds, who are rarely included in U.S. studies of GWG. ${ }^{29}$

Our results are consistent with previous studies demonstrating an association between excessive GWG in a single pregnancy and increased long-term maternal weight. ${ }^{5,8,33}$ Few studies to date have sought to incorporate weight gain over multiple pregnancies. ${ }^{12,13}$ Cohen et al. found that the prevalence of obesity at age 40 increased with the number of excessive GWG pregnancies in a nationally representative cohort. $^{12}$ Consistent with our study, the ordering of GWG adequacy across pregnancies did not affect the prevalence of obesity. Using the same cohort as the Cohen analysis, Abrams et al. ${ }^{13}$ estimated the impact of eliminating excessive GWG on incident obesity at a population level. Their analysis found that intervening in either the first or second pregnancy could significantly reduce the prevalence of midlife obesity among mothers.

The interpregnancy period is also of interest in understanding the impact of GWG across multiple pregnancies. Excessive GWG is positively correlated with increased postpartum weight retention. ${ }^{34-36}$ In addition, much of the effect of GWG on long-term maternal weight may be mediated through short-term postpartum weight retention. ${ }^{33}$ However, GWG adequacy remains an important opportunity for prevention, as the amount of weight retained postpartum depends on the amount gained during pregnancy. Further research is necessary to clarify the role of pregnancy complications. In our analysis, the number of pregnancies with hypertensive disorder was significantly associated with midlife BMI in fully adjusted models, while gestational

Adjusted $\mathrm{OR}$ and $95 \% \mathrm{Cl}$ of Midlife Obesity

FIG. 3. Adjusted ORs and 95\% confidence intervals of midlife obesity. Model adjusted for variables shown as well as study site, age at outcome measure, race/ethnicity, education, smoking, adolescent BMI, difficulty paying for basics, menopausal status, stress score, caloric intake, physical activity score, years since last birth, and age first pregnant. $\mathrm{OR}$, odds ratio.

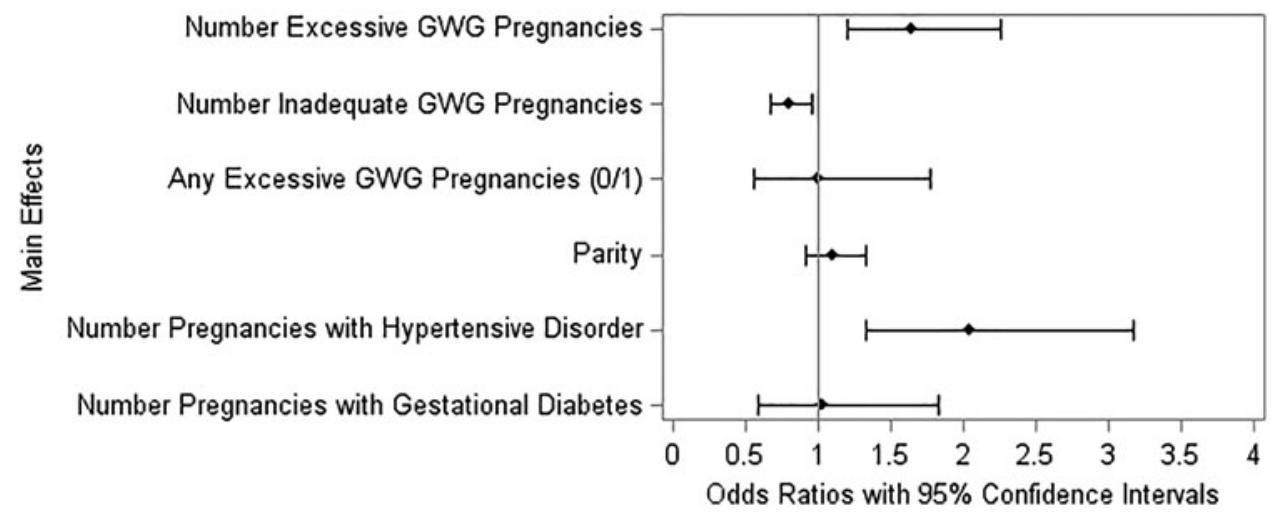




\section{Odds Ratio and $95 \% \mathrm{Cl}$ of Midlife Obesity by GWG Adequacy in the Last Pregnancy, Adjusted for GWG Adequacy of Prior Pregnancies Among Women with 1 to 3 Births $(n=1027)$}

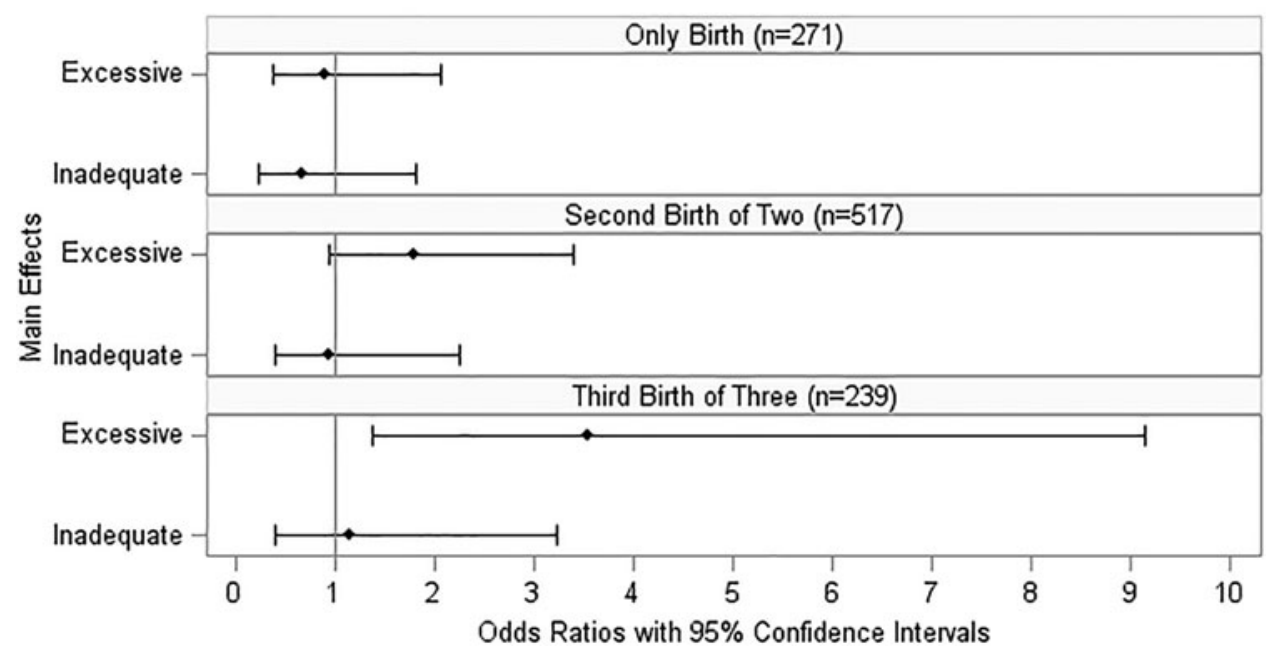

FIG. 4. ORs and 95\% confidence intervals of midlife obesity by GWG adequacy in the last pregnancy, adjusted for GWG adequacy of prior pregnancies, among women with one to three births. Model adjusted for GWG adequacy of previous pregnancy(ies), number of pregnancies with hypertensive disorder, number of pregnancies with gestational diabetes, study site, age at outcome measure, race/ethnicity, education, smoking, adolescent BMI, difficulty paying for basics, menopausal status, stress score, caloric intake, physical activity score, years since last birth, and age first pregnant. diabetes was not. This finding should be investigated further in studies with more precise measures of pregnancy complications. In addition, the association between GWG and midlife weight may differ for women with preterm births. While we excluded women with a history of preterm birth, work by McClure et al. observed an association between excessive GWG in a single pregnancy and later maternal weight in a cohort that oversampled for small-for-gestationalage and preterm births. ${ }^{9}$ Future research measuring GWG across multiple pregnancies, including those with complications and preterm delivery, would add to our understanding of the topic in a broader population of women.

Our study has several strengths. As noted, the ability to measure reproductive characteristics for all of our participants' births is unique in the literature. We were also able to capture GWG adequacy. Adequacy, which incorporates prepregnancy BMI, is a better representation of the health impacts of pregnancy weight compared to GWG amount alone. The use of adequacy also allowed us to adjust for inadequate GWG, so that adjusted models do not conflate insufficient gain with our definition of healthy gain. In addition, we had clinical measurement for the outcome of midlife BMI, rather than relying on self-reported weight and height. Finally, our analysis leveraged a key strength of SWAN, the depth of descriptive covariates available for this cohort. The collection of high-quality measures of lifestyle characteristics, including diet, physical activity, and stress, enabled us to adjust for a wide range of potentially confounding characteristics.

Limitations of this study include the retrospective exposure measurement and loss to follow-up in the cohort. Our measure of GWG relied on retrospective self-report collected an average of 30 years after the last birth. Previous research suggests that women often underestimate prepregnancy weight and overestimate GWG. ${ }^{37}$ Therefore, the prevalence of excessive GWG may be overestimated in studies relying on self-recall. Misclassification of women with adequate GWG as having excessive GWG could bias estimates toward the null. A metaanalysis by Headen et al. found moderate misclassification of GWG adequacy in studies relying on maternal recall of weight. Among studies measuring weight characteristics years after birth, mean deviation from true values was less than $1 \mathrm{~kg}$. However, the magnitude of error varied widely and was significantly greater among women with higher BMIs and those of minority race/ethnicity. In our data, repeated measures of self-reported GWG taken an average of 15.5 years apart showed moderate reliability, and the mean difference did not vary by obesity status. Moreover, the primary results were robust in sensitivity analyses. Smaller correlations between the two reports for participants with 5+ births may reflect increased difficulty recalling weight gain for each individual pregnancy. Despite known error, maternal recall has been used often in the literature as a practical measure to capture reproductive history. Collecting data prospectively for our hypothesis would be challenging, given the long time period between exposures in pregnancy and midlife outcomes.

Second, the loss of participants between the baseline visit and follow-up visit 13 was differential. Women who were excluded from this analysis due to missing data were different from included participants by several attributes. We sought to address this by adjusting for characteristics predictive of loss to follow-up such as stress, physical activity, and difficulty paying for basics. ${ }^{38}$ However, it is possible that our estimates include selection bias due to loss to follow-up.

Our results contribute to growing evidence that excessive GWG impacts long-term maternal health. The implications of these findings are particularly relevant in light of recent research that questions the strength of association of GWG adequacy with gestational and birth outcomes. ${ }^{39}$ Interventions to promote adequate GWG serve as maternal obesity prevention, in addition to impacts on birth and perinatal outcomes. Because over 90 percent of pregnant women receive some prenatal care in the United States, ${ }^{40}$ pregnancy can be seen as an important opportunity to protect women's long-term health.

\section{Conclusions}

In summary, our study found that each additional birth with excessive GWG was associated with an increase in mean maternal BMI at midlife. The cumulative effect we observed 
over multiple pregnancies implies that prevention of excessive GWG at any point in a woman's reproductive history can have an impact on long-term health. This highlights the importance of clinical counseling about healthy weight gain during pregnancy as an approach to obesity prevention. Studies with clinical measures of GWG should be conducted to confirm our findings. Further research could inform public health strategies for obesity prevention, as well as the clinical approach to maternal weight gain.

\section{Acknowledgments}

The content of this article is solely the responsibility of the authors and does not necessarily represent the official views of the NIA, NINR, or ORWH, or the NIH.

Clinical Centers: University of Michigan, Ann ArborSiobán Harlow, PI 2011-present, MaryFran Sowers, PI 1994 2011; Massachusetts General Hospital, Boston, MA-Joel Finkelstein, PI 1999-present; Robert Neer, PI 1994-1999; Rush University, Rush University Medical Center, Chicago, IL-Howard Kravitz, PI 2009-present; Lynda Powell, PI 1994-2009; University of California, Davis/Kaiser-Ellen Gold, PI; University of California, Los Angeles-Gail Greendale, PI; Albert Einstein College of Medicine, Bronx, NY-Carol Derby, PI 2011-present, Rachel Wildman, PI 2010-2011; Nanette Santoro, PI 2004-2010; University of Medicine and Dentistry-New Jersey Medical School, Newark-Gerson Weiss, PI 1994-2004; and the University of Pittsburgh, Pittsburgh, PA—Karen Matthews, PI.

NIH Program Office: National Institute on Aging, Bethesda, MD - Chhanda Dutta 2016 — present; Winifred Rossi 2012-2016; Sherry Sherman 1994-2012; Marcia Ory 19942001; National Institute of Nursing Research, Bethesda, MD_Program Officers.

Central Laboratory: University of Michigan, Ann ArborDaniel McConnell (Central Ligand Assay Satellite Services).

Coordinating Center: University of Pittsburgh, Pittsburgh, PA-Maria Mori Brooks, PI 2012-present; Kim SuttonTyrrell, PI 2001-2012; and New England Research Institutes, Watertown, MA—Sonja McKinlay, PI 1995-2001.

Steering Committee: Susan Johnson, Current Chair; Chris Gallagher, Former Chair.

We thank the study staff at each site and all the women who participated in SWAN.

\section{Author Disclosure Statement}

No competing financial interests exist.

\section{Funding Information}

The Study of Women's Health Across the Nation (SWAN) has grant support from the National Institutes of Health (NIH), DHHS, through the National Institute on Aging (NIA), the National Institute of Nursing Research (NINR), and the NIH Office of Research on Women's Health (ORWH) (Grants U01NR004061, U01AG012505, U01AG012535, U01A G012531, U01AG012539, U01AG012546, U01AG012553, U01AG012554, and U01AG012495).

\section{Supplementary Material}

Supplementary Table S1
Supplementary Table S2

Supplementary Table S3

Supplementary Table S4

Supplementary Table S5

Supplementary Table S6

Supplementary Table S7

\section{References}

1. Prevalence of Obesity Among Adults and Youth, United State 2011-2014. In: National Center for Health Statistics Data Brief No. 219: Centers for Disease Control and Prevention, 2015.

2. Ebbert JO, Elrashidi MY, Jensen MD. Managing overweight and obesity in adults to reduce cardiovascular disease risk. Curr Atheroscler Rep 2014;16:445.

3. Huang TT, Drewnosksi A, Kumanyika S, Glass TA. A systems-oriented multilevel framework for addressing obesity in the 21st century. Prev Chronic Dis 2009;6:A82.

4. Deputy NP, Sharma AJ, Kim SY, Hinkle SN. Prevalence and characteristics associated with gestational weight gain adequacy. Obstet Gynecol 2015;125:773-781.

5. Nehring I, Schmoll S, Beyerlein A, Hauner H, von Kries R. Gestational weight gain and long-term postpartum weight retention: A meta-analysis. Am J Clin Nutr 2011;94:12251231.

6. Krukowski RA, Bursac Z, McGehee MA, West D. Exploring potential health disparities in excessive gestational weight gain. J Womens Health (Larchmt) 2013;22:494500 .

7. Jarman M, Yuan Y, Pakseresht M, Shi Q, Robson PJ, Bell RC. Patterns and trajectories of gestational weight gain: A prospective cohort study. CMAJ Open 2016;4:E338-E345.

8. Widen EM, Whyatt RM, Hoepner LA, et al. Excessive gestational weight gain is associated with long-term body fat and weight retention at $7 \mathrm{y}$ postpartum in African American and Dominican mothers with underweight, normal, and overweight prepregnancy BMI. Am J Clin Nutr 2015;102:1460-1467.

9. McClure CK, Catov JM, Ness R, Bodnar LM. Associations between gestational weight gain and BMI, abdominal adiposity, and traditional measures of cardiometabolic risk in mothers 8 y postpartum. Am J Clin Nutr 2013;98: 1218-1225.

10. Davis EM, Babineau DC, Wang X, et al. Short interpregnancy intervals, parity, excessive pregnancy weight gain and risk of maternal obesity. Matern Child Health J 2014;18:554-562.

11. Mamun AA, Kinarivala M, O'Callaghan MJ, Williams GM, Najman JM, Callaway LK. Associations of excess weight gain during pregnancy with long-term maternal overweight and obesity: Evidence from 21 y postpartum follow-up. Am J Clin Nutr 2010;91:1336-1341.

12. Cohen AK, Chaffee BW, Rehkopf DH, Coyle JR, Abrams B. Excessive gestational weight gain over multiple pregnancies and the prevalence of obesity at age 40 . Int $\mathrm{J}$ Obes (Lond) 2014;38:714-718.

13. Abrams B, Coyle J, Cohen AK, et al. Excessive gestational weight gain and subsequent maternal obesity at age 40: A hypothetical intervention. Am J Public Health 2017;107: 1463-1469.

14. Avis NE, Crawford SL, Greendale G, et al. Duration of menopausal vasomotor symptoms over the menopause transition. JAMA Intern Med 2015;175:531-539. 
15. Thurston RC, Sowers MR, Sternfeld B, et al. Gains in body fat and vasomotor symptom reporting over the menopausal transition: The Study of Women's Health Across the Nation. Am J Epidemiol 2009;170:766-774.

16. Randolph JF, Jr., Sowers M, Gold EB, et al. Reproductive hormones in the early menopausal transition: Relationship to ethnicity, body size, and menopausal status. J Clin Endocrinol Metab 2003;88:1516-1522.

17. Sowers MFR, Crawford SL, Sternfeld B, et al. SWAN: A multicenter, multiethnic, community-based cohort study of women and the menopausal transition. In: Lobo RK, Marcus R, eds. Menopause: biology and pathobiology. San Diego: Academic Press, 2000:175-188.

18. Bodnar LM, Hutcheon JA, Parisi SM, Pugh SJ, Abrams B. Comparison of gestational weight gain $\mathrm{Z}$-scores and traditional weight gain measures in relation to perinatal outcomes. Paediatr Perinat Epidemiol 2015;29:11-21.

19. Hutcheon JA, Platt RW, Abrams B, Himes KP, Simhan HN, Bodnar LM. Pregnancy weight gain charts for obese and overweight women. Obesity (Silver Spring) 2015;23: 532-535.

20. Rasmussen KM, Yaktine AL. Weight Gain During Pregnancy: Reexamining the Guidelines. Washington, DC: Institute of Medicine, National Academies Press, 2009.

21. World Health Organization. The Asia-Pacific Perspective: Redefining obesity and its treatment. Sydney: Health Communications Australia, 2000.

22. Shiwaku K, Anuurad E, Enkhmaa B, et al. Predictive values of anthropometric measurements for multiple metabolic disorders in Asian populations. Diabetes Res clin Pract 2005;69:52-62.

23. Nguyen TT, Adair LS, He K, Popkin BM. Optimal cutoff values for overweight: Using body mass index to predict incidence of hypertension in 18- to 65-year-old Chinese adults. J Nutr 2008;138:1377-1382.

24. Anuurad E, Shiwaku K, Nogi A, et al. The new BMI criteria for Asians by the regional office for the western pacific region of WHO are suitable for screening of overweight to prevent metabolic syndrome in elder Japanese workers. J Occup Health 2003;45:335-343.

25. Morisaki N, Nagata C, Jwa SC, et al. Pre-pregnancy BMIspecific optimal gestational weight gain for women in Japan. J Epidemiol 2017;27:492-498.

26. Razak F, Anand SS, Shannon H, et al. Defining obesity cut points in a multiethnic population. Circulation 2007;115: 2111-2118.

27. Palaniappan LP, Wong EC, Shin JJ, Fortmann SP, Lauderdale DS. Asian Americans have greater prevalence of metabolic syndrome despite lower body mass index. Int $\mathbf{J}$ Obes (Lond) 2011;35:393-400.

28. Wie JH, Park IY, Namkung J, Seo HW, Jeong MJ, Kwon JY. Is it appropriate for Korean women to adopt the 2009 Institute of Medicine recommendations for gestational weight gain? PLoS One 2017;12:e0181164.
29. Khanolkar AR, Hanley GE, Koupil I, Janssen PA. 2009 IOM guidelines for gestational weight gain: How well do they predict outcomes across ethnic groups? Ethn Health 2017:1-16.

30. Block G, Hartman AM, Dresser CM, Carroll MD, Gannon J, Gardner L. A data-based approach to diet questionnaire design and testing. Am J Epidemiol 1986;124:453-469.

31. Sternfeld B, Ainsworth BE, Quesenberry CP. Physical activity patterns in a diverse population of women. Prev Med 1999;28:313-323.

32. Cleveland WS, Devlin SJ. Locally weighted regression: An approach to regression analysis by local fitting. J Am Stat Assoc 1988;83:596-610.

33. Kirkegaard H, Stovring H, Rasmussen KM, Abrams B, Sorensen TI, Nohr EA. How do pregnancy-related weight changes and breastfeeding relate to maternal weight and BMI-adjusted waist circumference $7 \mathrm{y}$ after delivery? Results from a path analysis. Am J Clin Nutr 2014;99:312319.

34. Mannan M, Doi SA, Mamun AA. Association between weight gain during pregnancy and postpartum weight retention and obesity: A bias-adjusted meta-analysis. Nutr Rev 2013;71:343-352.

35. Butte NF, Ellis KJ, Wong WW, Hopkinson JM, Smith EO. Composition of gestational weight gain impacts maternal fat retention and infant birth weight. Am J Obstet Gynecol 2003;189:1423-1432.

36. Lederman SA, Paxton A, Heymsfield SB, Wang J, Thornton J, Pierson RN, Jr. Body fat and water changes during pregnancy in women with different body weight and weight gain. Obstet Gynecol 1997;90(Pt 1):483-488.

37. Headen I, Cohen AK, Mujahid M, Abrams B. The accuracy of self-reported pregnancy-related weight: A systematic review. Obes Rev 2017;18:350-369.

38. Little RJ, Rubin DB. Statistical analysis with missing data, vol. 333. Hoboken, NJ: John Wiley \& Sons, 2014.

39. Voerman E, Santos S, Inskip H, et al. Association of gestational weight gain with adverse maternal and infant outcomes. JAMA 2019;321:1702-1715.

40. Martin JA, Hamilton BE, Osterman MJK, Driscoll AK, Drake P. Births: Final data for 2017. Natl Vital Stat Rep 2018;67:1-50.

\author{
Address correspondence to: \\ Franya Hutchins, MS, MA \\ Department of Epidemiology \\ Graduate School of Public Health \\ University of Pittsburgh \\ 130 De Soto Street \\ Pittsburgh, PA 15261 \\ E-mail: frh16@pitt.edu
}

